\title{
OBSERVATIONS OF THE CELESTIAL MOTION OF THE EARTH'S POLE
}

\author{
M. FEISSEL AND A.-M. GONTIER \\ Central Bureau of IERS, Paris Observatory, CNRS/URA1125 \\ 61, avenue de l'Observatoire \\ 75014 Paris, France
}

\begin{abstract}
The accurate observation of the celestial motion of the Earth's pole requires careful connection of the VLBI observables with quantities and reference frames related to the theoretical description of precession-nutation models. The analysis of the derived celestial pole offsets beyond the semi-empirical IERS(1996) precession-nutation model shows variability of some components, such as the seasonal term and $\mathrm{a} \sim 430$ day term.
\end{abstract}

\section{Reference systems, conceptual definitions and VLBI observables}

The celestial motion of the Earth's pole is observed in the International Celestial Reference System (ICRS, Arias et al., 1995) recommended for use by the IAU starting with 1 January 1998. The realization of this system, the International Celestial Reference Frame (ICRF), is described in Ma et al. (1997). A description of the consequences in the current astronomical procedures induced by this change is given by Kovalevsky and McCarthy (1997).

The observations consist of time series of VLBI-derived coordinates of the Celestial Ephemeris Pole (CEP) in the ICRS. The CEP is by convention a pole that has no diurnal motion either in space or in the Earth. The IAU 1980 Theory of Nutation (Seidelmann, 1982) models the periodic celestial motion of the CEP relative to the mean pole at J2000.0 corresponding to the 1976 IAU precession model (Lieske et al., 1976). In VLBI data analysis, the IAU 1976-1980 models are used as a prioris and the deviation of the actual celestial direction of the CEP is estimated from $24 \mathrm{~h}$ observing sessions. The corresponding parameters are the so-called celestial pole offsets, $\mathrm{d} \psi$ and $\mathrm{d} \varepsilon$. As the VLBI data analysis is referred to the ICRS while the celestial pole offsets are referred to the mean pole at $\mathrm{J} 2000.0$, the time series of the celestial pole offsets include a constant term that is due to the fact that the mean pole at J2000.0 is not in coincidence with the ICRS pole. The shift, shown in Figure 1, is estimated to be $17.24 \pm 0.01$ mas in the direction $12 \mathrm{~h}$ and $5.1 \pm 0.01$ mas in the direction 18h (IERS 1997). For reference, the FK5 pole position (see Kovalevsky and McCarthy, 1997) is also shown; the FK5 pole was meant to be a realization of the mean pole at J2000.0.

Very Long Baseline Interferometry (VLBI) is the only technique that can provide the tie to a celestial reference system, a terrestrial reference system and the Earth orientation parameters simultaneously. The modeling of VLBI observables, the delay and the delay rate, is made in the framework of General Relativity. It includes the transformation from terrestrial to celestial coordinate systems (McCarthy, 1996). Mass redistribution in the solid Earth and the oceans by global tides affect both universal time and polar motion and modify the Earth's orientation. Although these effects are ignored in the context of the definition of the CEP, they have to be considered. Diurnal and semi-diurnal variations in polar motion and UT1 are therefore taken into account (see Herring, 1991). The major sets of VLBI analysis software have been intensively tested and intercompared. They are consistently implemented within $1 \mathrm{ps}$ for the delay and $1 \mathrm{fs} / \mathrm{s}$ for the delay rate, one order of magnitude better than the observations (Jacobs et al., 1997). 


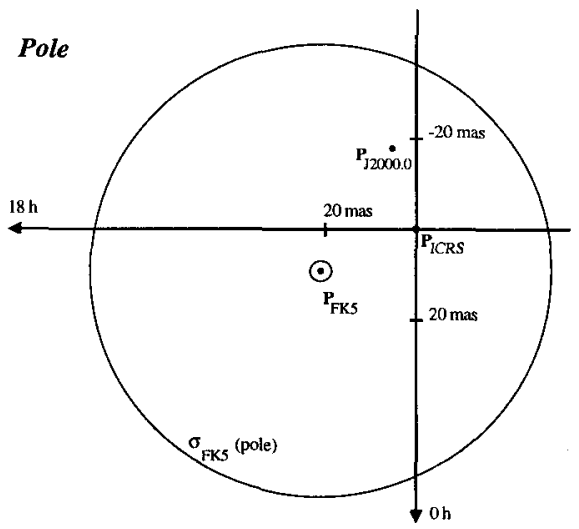

- Direction of the mean pole at

J2000.0 in the ICRS, determined

by VLBI and LLR (uncertainty 0.1 mas)

- Direction of the FK5 pole in the ICRS

determined by comparison with Hipparcos (uncertainty 2.3 mas at J2000.0).

Large circle: original uncertainty of the FK5 pole ( 50 mas).

Figure 1. Physical and conventional directions in the International Celestial Reference System, ICRS.

\section{Analysis of celestial pole offsets time series, 1984-1996}

The series analyzed here, EOP(GSFC) 97 R 01, was obtained by Ma et al. (1997), over 1979-1996 from all existing VLBI observations. The time series of celestial pole offsets derived from VLBI observations show evidence of errors in the IAU (1980) Theory of Nutation and in the IAU (1976) Precession that are to a large extent corrected in the IERS Conventions (1996) semi-empirical model, as described by Mathews and Herring (1997). The latter model accounts for $99.4 \%$ of the variance of the observed differences with the conventional IAU models. However, the signal remaining after this correction is still one order of magnitude larger than the noise of a single observation ( \pm 0.1 mas in 1996). The remaining variance is mainly localized in high frequencies as illustrated in Table 1.

TABLE 1. Precision and noise level of VLBI celestial pole results, 1984-1996 EOP(GSFC) 97 R 01 (Ma et al., 1997) - IERS Conventions (1996) Unit: $0.001 "$

\begin{tabular}{|c|c|c|c|c|c|c|c|c|c|c|}
\hline \multirow[t]{3}{*}{ Years } & \multirow{2}{*}{\multicolumn{2}{|c|}{$\begin{array}{c}\text { uncertainty } \\
\text { of a single value }\end{array}$}} & \multicolumn{8}{|c|}{ weighted root mean square residual } \\
\hline & & & \multicolumn{2}{|c|}{ all periods } & \multicolumn{2}{|c|}{$\begin{array}{c}\text { with } 433 \mathrm{~d} \text { term } \\
\text { estimated }\end{array}$} & \multicolumn{2}{|c|}{$\begin{array}{c}\text { for periods } \\
\text { longer than } 30 \mathrm{~d}\end{array}$} & \multicolumn{2}{|c|}{$\begin{array}{c}\text { for periods } \\
\text { shorter than } 30 \mathrm{~d}\end{array}$} \\
\hline & $\psi$ & $\varepsilon$ & $\psi$ & $\varepsilon$ & $\psi$ & $\varepsilon$ & $\psi$ & $\varepsilon$ & $\psi$ & $\epsilon$ \\
\hline $1984-85$ & 1.04 & 0.42 & 1.40 & 1.17 & 1.11 & 1.14 & 0.67 & 0.25 & 0.89 & 1.11 \\
\hline $1985-86$ & 0.76 & 0.30 & 1.38 & 1.59 & 1.13 & 1.31 & 0.54 & 0.22 & 0.99 & 1.29 \\
\hline $1986-87$ & 0.71 & 0.25 & 1.46 & 1.46 & 1.11 & 1.34 & 0.48 & 0.20 & 1.00 & 1.32 \\
\hline $1987-88$ & 0.67 & 0.26 & 1.49 & 1.59 & 1.24 & 1.49 & 0.43 & 0.21 & 1.17 & 1.48 \\
\hline $1988-89$ & 0.54 & 0.23 & 1.69 & 1.68 & 1.45 & 1.42 & 0.46 & 0.16 & 1.38 & 1.41 \\
\hline $1989-90$ & 0.41 & 0.17 & 1.43 & 1.57 & 1.36 & 1.33 & 0.38 & 0.14 & 1.31 & 1.32 \\
\hline $1990-91$ & 0.34 & 0.14 & 1.47 & 1.47 & 1.34 & 1.26 & 0.35 & 0.13 & 1.29 & 1.25 \\
\hline $1991-92$ & 0.30 & 0.13 & 1.42 & 1.30 & 1.31 & 1.15 & 0.33 & 0.13 & 1.27 & 1.14 \\
\hline $1992-93$ & 0.26 & 0.11 & 1.34 & 1.29 & 1.18 & 1.15 & 0.31 & 0.13 & 1.14 & 1.14 \\
\hline $1993-94$ & 0.19 & 0.08 & 0.98 & 1.19 & 0.82 & 1.01 & 0.25 & 0.12 & 0.78 & 1.00 \\
\hline 1994-95 & 0.15 & 0.06 & 0.76 & 0.74 & 0.63 & 0.66 & 0.21 & 0.09 & 0.59 & 0.65 \\
\hline $1995-96$ & 0.16 & 0.06 & 0.80 & 0.84 & 0.69 & 0.79 & 0.23 & 0.11 & 0.65 & 0.78 \\
\hline
\end{tabular}

The residual motion not accounted for by the IERS Conventions (1996) model is plotted on 


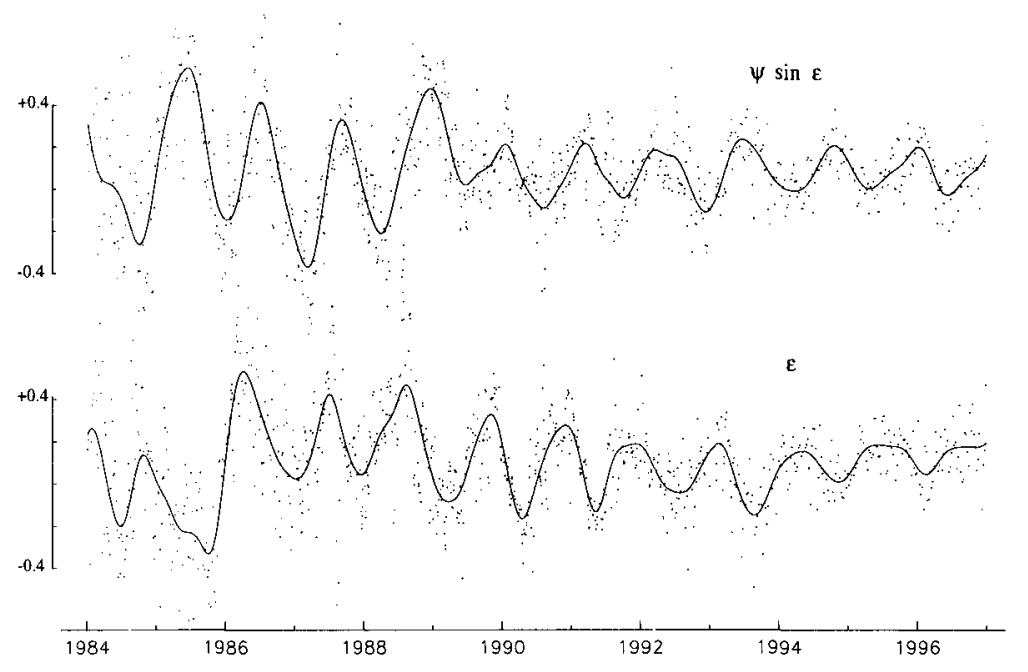

Figure 2. Variations in the celestial pole offsets not accounted for by the IERS Conventions (1996) precession-nutation model. The dots are the individual determinations. The solid line is a Vondrak smoothing that keeps periods longer than 300 days. Unit: 0.001 "

Figure 2. The oscillation shown by the solid line is generally considered to be the effect of the Free Core Nutation (FCN). The theoretical predictions of the FCN period span a few days around 433 days, and active research is made by different groups to explain that its amplitude seems to vary with time, or with the time interval considered (e.g. Dehant et al., 1997, and other papers in this JD proceedings). If one considers a unique FCN period (433 days) over 1984-96, the mean amplitudes (in $\psi \sin \varepsilon$ or $\varepsilon$ ) determined over the two-year intervals of Table 1 range from 0.32 to 0.06 mas, with formal uncertainties of 0.07 mas at the beginning and 0.03 mas at the end of the period covered.

The IERS Conventions (1996) precession-nutation model gives a constant amplitude for the 365.22 day term. While the rigid-Earth term has only in-phase components, the observed annual nutation has statistically significant out-of-phase components. In addition, both the in-phase and out-of-phase components have significant variations from year to year, as shown on Figure 3 . These variations are ascribed to atmospheric effects. 

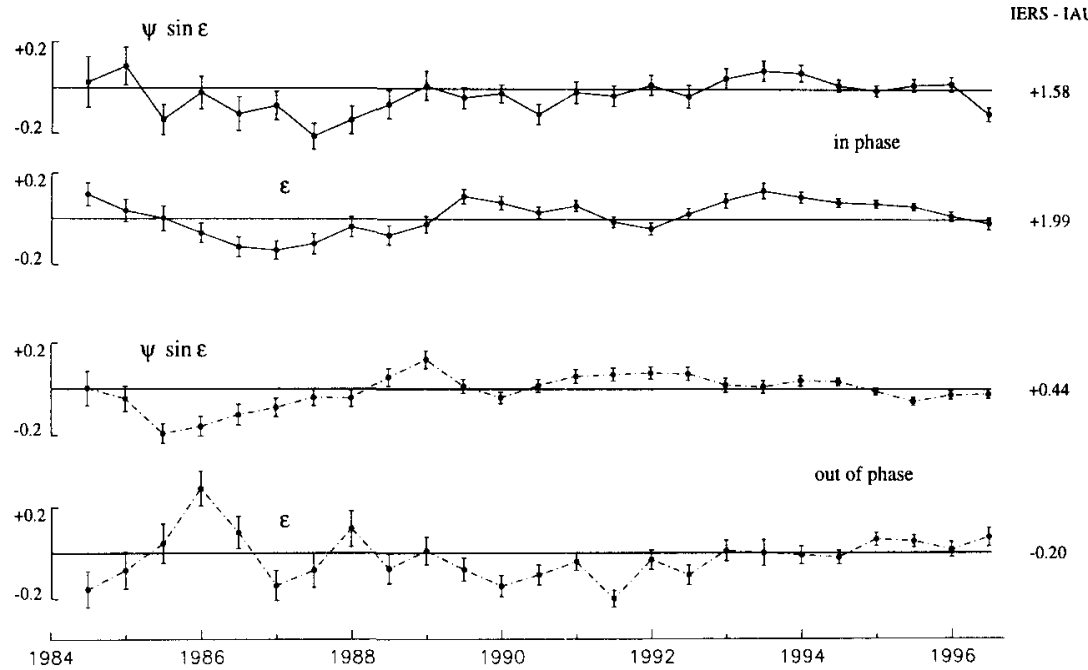

Figure 3. Year to year variations in the components of the 365.22 day nutation term with their standard deviations, relative to the mean IERS corrections, given on right of the figure. The plotted values are determined over one-year intervals, shifted by 0.5 year. Unit: 0.001 "

\section{References}

Arias, E.F., Charlot, P., Feissel, M. and Lestrade, J.-F. (1995) The extragalactic reference system of the International Earth Rotation Service, ICRS, Astron. Astrophys., 303, pp. 604-608.

Dehant, V., Feissel, M., Defraigne, P., Roosbeek, F. and Souchay, J. (1997) Could the energy near the FCN and the FICN be explained by luni-solar or atmospheric forcing?, Geophys. J. Int., 130, pp. 535-546.

Herring, T.A. (1991) The ZMOA-1990 nutation series, in "Reference Systems", Proceedings of the IAU Colloquium 127, eds A. Hugues, C. Smith, G. Kaplan, U.S. Naval Observatory, Washington, pp. 175-166.

IERS (1997) 1996 IERS Annual Report, Observatoire de Paris, Paris, pp. II-38.

Jacobs, C.S., Sovers, O.J., Gordon, D., Ma, C. and Gontier, A.-M. (1997) The accuracy of the ICRF: an intercomparison of VLBI analysis software, JD 7 this volume.

Kovalevsky, J. and McCarthy, D.D. (1997) Astronomical effects of current changes in fundamental astrometric references, JD 3 this volume.

Lieske, J.H., Lederle, T., Fricke, W. and Morando, B. (1977) Expressions for the Precession Quantities Based upon the IAU (1976) System of Astronomical Constants, Astron. Astrophys., 58, pp. 1-16.

Ma, C., Arias, E.F., Eubanks., T.M., Fey, A.L., Gontier, A.-M., Jacobs, C.S. and Sovers, O.J. (1997) Formation of the ICRF, JD 7 this volume.

Ma, C. and Baver, K.D. (1997) Site Positions and Velocities, and Earth Orientation Parameters from the NASA Space Geodesy Program-GSFC: Solutions GLB1069 and GLB1070,1996 IERS Annual Report, description available under anonymous ftp: hpiers.obspm.fr file iers/annreports/1996AR/gsfc.r

Mathews, P.M and Herring, T.A. (1997) Observational and Theoretical Modeling of Nutation, JD 3, this volume.

McCarthy, D.D. (ed) (1996), IERS Conventions, IERS technical Note, 21, Observatoire de Paris, Paris, pp. 20-39.

Seidelmann, P.K. (1982) 1980 IAU Theory of Nutation: The Final Report of the IAU Working Group on Nutation, Celestial Mechanics, 27, pp. 79-106. 